\title{
3D IMAGE BASED GEOMETRIC DOCUMENTATION OF A MEDIEVAL FORTRESS
}

\section{S. Tapinaki ${ }^{1}$, M. Skamantzari ${ }^{1}$, R. Chliverou ${ }^{1}$, V. Evgenikou ${ }^{2}$, A.M. Konidi ${ }^{3}$, E. Ioannatou ${ }^{3}$, A. Mylonas ${ }^{4}$, A. Georgopoulos ${ }^{1}$}

\author{
${ }^{1}$ National Technical University of Athens, School of Rural and Surveying Engineering, Lab of Photogrammetry, Zografou Campus, \\ Heroon Polytechniou 9, 15780, Zografou, Athens, Greece \\ sevi@survey.ntua.gr,mskamantz@central.ntua.gr, regina@survey.ntua.gr,drag@central.ntua.gr \\ ${ }^{2}$ Rural and Surveying Engineer, MSc Protection of Monuments, Athens, Greece \\ vevge@ hotmail.com \\ ${ }^{3}$ Architect Engineer, MSc Protection of Monuments, Athens, Greece \\ amkonidi@gmail.com, eva.ioannatou@gmail.com \\ ${ }^{4}$ Architect Engineer, Athens, Greece \\ mylonas.apo@gmail.com
}

\section{Commission II}

KEY WORDS: 3D model, Orthophoto, UAV, Laser Scanning, Photogrammetry

\begin{abstract}
:
The detailed and thorough documentation of monuments is a rather complex process that requires the application of the best available state of the art techniques in order to preserve, restore, promote and make cultural heritage accessible to the public. This paper presents the 3D Geometric Documentation of a part of the medieval fortress of Chios, focussing in particular on the practical challenges which the object presented. The case study is a part of the fortified construction, consisting of a bastion, a watch tower on top of this bastion and a significant part of its walls with a surface of about $1053 \mathrm{~m}^{2}$ in total. The goal of the survey was to produce an accurate 3D detailed textured model and a series of coloured orthophotos and 2D vector drawings. The documentation methods employed included closerange automated photogrammetry and image-based modelling, terrestrial laser scanning and topographic surveys, an ideal combination of methods.
\end{abstract}

\section{INTRODUCTION}

Numerous efforts and milestones have been made so far concerning the precise and realistic 3D digitisation of monuments and archaeological sites in order to achieve the necessary accuracy, thoroughness and trueness for the documentation's products and applications. The products of the 3D digitisation of cultural heritage enable archaeologists, engineers, architects, cultural heritage experts and stakeholders in general to approach their case studies and make decisions in a complete and efficient way as far as the approaches of documentation, restoration and enhancement of monuments are concerned.

Three-dimensional Geometric Documentation is the essential way of recording the present state of monuments, as it has evolved through time and supply the necessary metric and informative background to those who investigate the past, but also to those who care about the future of monuments. The main goal of the Geometric Documentation is to record the existing state, the position, size and shape of a monument or archaeological site at a particular moment in time (Tapinaki et al., 2005).

The 3D Geometric Documentation of a specific part of the Medieval Fortress of Chios was considered essential in order to generate the necessary products which are intended to be used by the local Archaeological Ephorate for restoration purposes. In particular, the goal of the survey was to produce the accurate $3 \mathrm{D}$ detailed textured model of a bastion, a watch tower and a significant part of the walls as well as a set of coloured orthophotos and 2D vector drawings at 1:25 and 1:40 scales, including the facades of the tower and the walls, the ground plan and two vertical sections. In addition, the development of bastion, believed to be a cylindrical surface, was required.

\section{THE MEDIEVAL FORTRESS OF CHIOS}

Chios is an island in the Eastern part of the Aegean Sea, approximately $220 \mathrm{~km}$ eastwards from Athens (Figure 1). The island has a long history and in the Medieval times Genovese conquerors occupied many islands in the area.

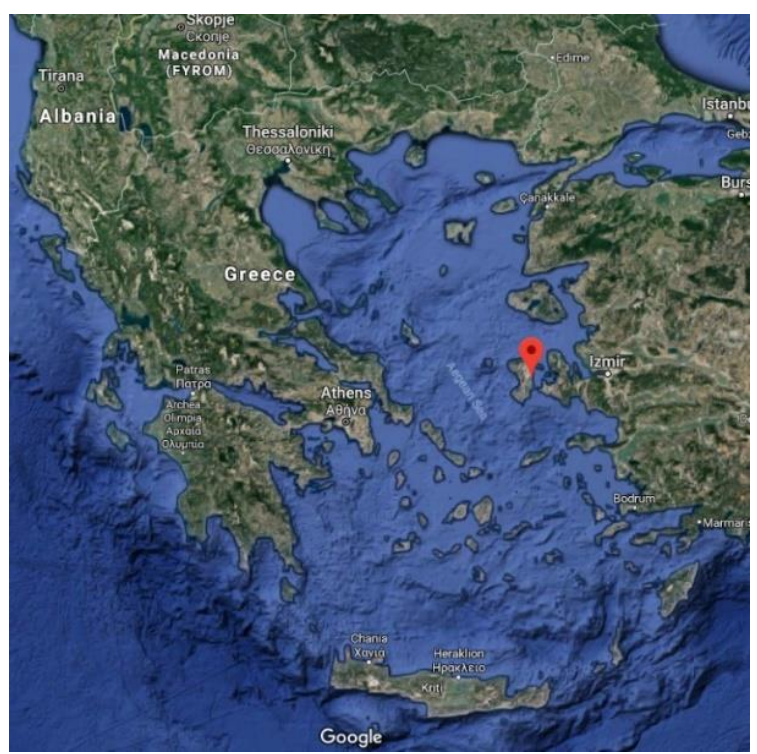

Figure 1: Location of Chios 
The fortress of Chios covers an area of $180,000 \mathrm{~m}^{2}$ and nowadays its walls include a residential complex with 650 inhabitants. It used to be the walled core of the town since the Genovese period, however there is evidence that the Castle had been inhabited since the Hellenistic years $\left(4^{\text {th }}\right.$ c. BC), as well as during the Roman period and the early byzantine years. Its walls both in land and at sea form an irregular pentagon, with strong bastions, eight of which are still preserved (Figure 2).

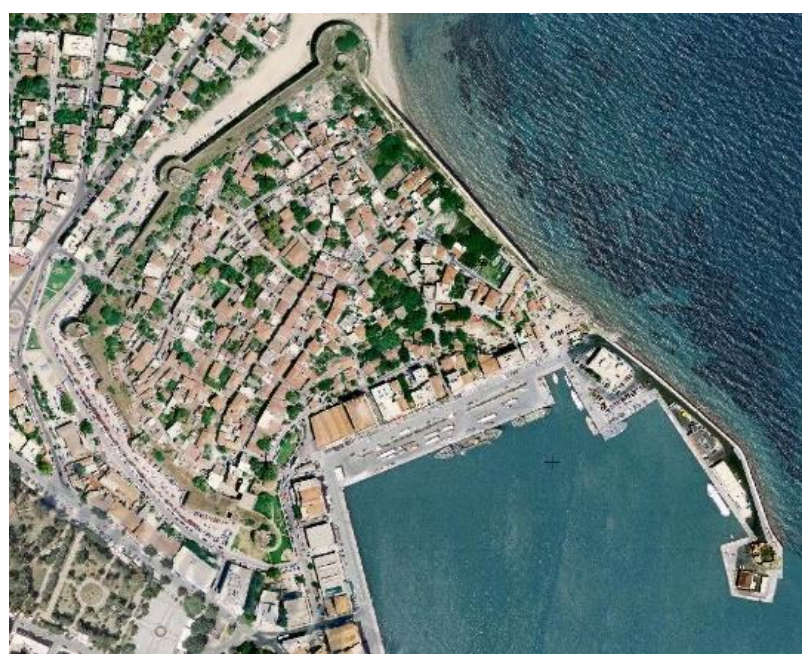

Figure 2: Aerial view of The Fortress and Port of Chios

The Greek Ministry of Culture designated the area of the Fortified City as a Monument of outstanding value (Law of 29.10.2001), due to the distinctive features of its tangible character and its intangible values that it acquired through the ages (Papathanassiou-Zuhrt, Doumi, 2006). The Archaeological Zone includes the south-eastern part of the City, which extends north-eastern of the Port of Chios. The Fortified City is one of the most significant monuments of Chios since it is the remaining evidence and testimony of the coexistence of different cultures and civilisations hosted at Chios through the ages.

The Fortress was many times destroyed and rebuilt and in the latest years major damage was caused by the Ottoman bombardments in 1828 and the earthquake of 1881 . The evolution of the artillery and the extended use of gun powder led to new elements in the defence systems and as a result the towers were turned to fortified, polygonal or semi-circular platforms which extended out of the main defence line. This evolution led to the formation of the bastions as seen today. Another important typological element of the fortress is the inclinations (scarpe) that can be found at the lower part of the walls. The cortine were coated by stone forming the scarpe, concluding to a cordone, a stone frieze type, jutting out of the wall, serving the purpose to prevent the enemy to put ladders on the wall (Figure 3) (Papathanassiou-Zuhrt, Doumi, 2006).

Since 1924, the Greek Ministry of Culture has taken measures to protect the monument and in the same year it designated the fortress as a protected archaeological and historical site. The restoration of the on-land part of the fortress which is the most prominent part of the monument, directly visible from the harbour and the city centre, began two years ago and has been completed at the end of 2018. The works involved mild architectural interventions which made the monument safe and accessible to the public.

The typology and development of fortifications through the ages give significant information about the structure, the component elements of any fortification function, the form, shape and use of them, so these elements should be taken into consideration when it comes to documentation in general. The typology of a monument and its architectural details play a fundamental role to the decisions and choices of the documentation and can even lead to the final products.

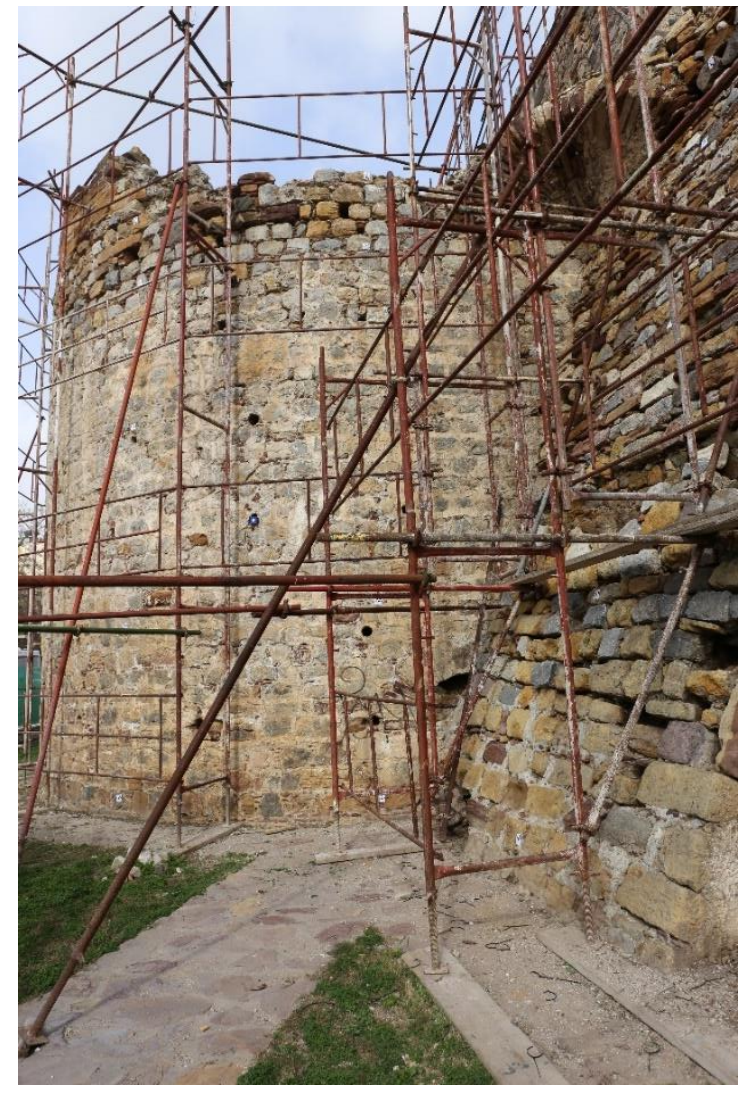

Figure 3: Detail of the area to be documented

\section{STATE-OF-THE-ART}

Documenting fortified constructions presents some challenges. Raster and vector products for documentation of fortification monuments can be used in a variety of applications; in studies of archaeological or architectural interest [Drap et al 2005, Ringle et al. 2005, Gianniou and Georgopoulos, 2007, Chiabrando et al., 2009, Vrettou and Georgopoulos, 2016], for designing maintenance and restoration work [Almagro-Gorbea and Orihuela Uzal, 2015, Kersten et al., 2015], structural analysis of constructions [Arias et al., 2007], digital reconstruction of damaged or destroyed monuments [Grussenmeyer and Yasmine, 2003, Rodriguez-Navarro and Verdiani, 2013] for historical interpretation, and for upgrading the touristic product of a region. An accurate and faithful 3D representation is an important stepping stone from which a variety of products and applications can be generated. The file size of a 3D model file and an orthophoto in comparison to a $2 \mathrm{D}$ plan reflects the additional amount of information included in raster products. Interpretation and exclusion of additional information is required in cooperation with the end users of the products.

When it comes to cultural heritage there is often the need for creating developments of curved, second order surfaces like cylindrical, conical, spherical, parabolic or ellipsoidal surfaces in order to have a better conception of the monument. There are many examples and applications that can be found in the literature concerning the geometric documentation of fortifications and the creation of developments derived from 
photogrammetric data, mainly in spherical domes and cylinders (Feltham 1990, Theodoropoulou 1996).

In 1999 the Hellenic Ministry of Culture in collaboration with the Laboratory of Photogrammetry of the NTUA undertook the detailed restitution and representation of the seaward castle of Chios (Ioannides and Potsiou, 1999). In this case there were certain developments among the final products, some of which represented simple curved surfaces which could be easily defined mathematically. However, the external side of the seaward tower of Chios presented some difficulties as far as the development is concerned since the façade could not be mathematically defined as a surface, making any adaptation rather uncertain, as well as the geometry of the structure consisted of more than one mathematically defined objects, which is rather common in monuments.

Another example of digital surface development is that of two Hellenistic Towers, the cylindrical tower of Drakanos on the island of Ikaria with an external diameter of $8-5 \mathrm{~m}$. and a height of $12 \mathrm{~m}$., and the conical tower of Ag, Petros on the island of Andros with a diameter of about $10 \mathrm{~m}$. at base level and a height of 19m. (Theodoropoulou et al., 2001). A single-image technique was applied to this case for the development of 3D geometrical surfaces to which analytical surfaces were fitted giving efficient and accurate solutions for architectural surveys.

In 2001 a method for cylindrical orthorectification and development of surfaces was applied on the case of the antique tower Laudun oppidum in the south of France and the towers of the Blandy-les-Tours Castle near Paris (Chazaly, 2001). In the case of the antique tower Laudun oppidum the cylindrical orthophoto was developed for the outside surface of the foundation and the elevation, while in the case of the towers of the Blandy-les-Tours a cartographic document was produced with the unwrapped orthophoto, the contour lines of the corresponding unwrapped DEM, the geometrical information about the cylindrical surface of projection and statistics about the 3D point cloud, as well as a document with all the cylindrical orthophotos in order to provide a coverage of all the unwrapped elevation of each tower. In this last case, it is worth mentioning that during the 3D documentation a systematic shifting of the centres of the measured cylinders was revealed, since the inside cylinders are tangent to the inside of the castle, whereas the outside surface seems to be a vertical cylinder.

Georgopoulos et al., 2002 refer to the detailed archaeological and artistic research of the Byzantine Monastery of Daphni in Athens, after a strong earthquake in 1999, where the recording of the magnificent mosaics of the main church, i.e. the Katholikon, at a very large scale, was considered necessary. For this task a specialized software has been developed in order to produce the raster developments of those mosaics which are constructed on developable, i.e. cylindrical or conical, surfaces.

Nowadays, different strategies can be followed, and various software can be used for the development of a second order surface, developable or not, in order to produce an orthoimage since SfM and MVS algorithms are mostly used for the 3D surface reconstruction from multiple digital images. Commercial software 3DReshaper and Geomagic Wrap, as well as opensource software Cloud Compare offer effective tools to calculate cylindrical and conical projection and unwrap the 3D mesh surface or the point cloud (Sammartano, Spanò, 2017).

The Agisoft Photoscan Pro v.1.4 allows to project orthomosaic on a cylindrical surface, avoiding severe distortions. The user defines the cylinder i.e. axis and radius, a perpendicular point from the $3 \mathrm{D}$ cloud to the cylinder's axis and agrees on the way that $\mathrm{x}$ and $\mathrm{y}$ coordinates of a point are defined on the cylinder (www.agisoft.com).

\section{METHODOLOGY}

Current technological advances, techniques and methods offer numerous different combinations for the 3D Geometric Documentation of monuments and archaeological sites depending on the level of complexity, the object's size, the accuracy of the products, the available equipment, the cost and the documentation scale of the project. Although, combining different methods has been proven the ideal solution for the geometric documentation in order to overcome the flaws of each technique and achieve the proper, accurate results.

The determination of the reference coordinate system that will be used is of utmost importance and must be done before any other work. The use of an arbitrary local coordinate reference system georeferenced with the minimum constraints will ensure the avoidance of deformations of the shape or size of the monument.

In the case of the medieval fortress of Chios it was decided to employ close-range automated photogrammetry and imagebased modelling, as well as terrestrial laser scanning and topographic surveys. In particular, the following equipment were used: An Integrated Total Station (Topcon GPT3003LN), a timeof-flight pulse-based 3D Laser Scanner (Leica Scanstation 10), two DSLR cameras (Canon EOS 6D and Canon 80D with 8$15 \mathrm{~mm}, 24 \mathrm{~mm}$ and $18-55 \mathrm{~mm}$ Lenses), and an Unmanned Aerial Vehicle (DJI Phantom 4 Pro).

\subsection{Data Acquisition}

Firstly, a trigonometric network was established comprised of 7 points from which the ground control points (GCPs) were measured, but also additional supportive measurements were performed. The network was set up in an arbitrary local coordinate reference system, which subsequently was georeferenced with the minimum constraints to avoid deformations of the shape or size of the monument due to the projection. The accuracy of the network was $0,9 \mathrm{~mm}$ and a total number of ----- GCPs was measured, as well as the necessary points for two vertical sections. In addition, the laser scanner targets were also determined in order to reference the point clouds to the same coordinate system.

This present case study was particularly challenging since dense scaffolding was set on site, in direct contact with the object to be documented, making both the data acquisition process and the data processing afterwards extremely challenging and time consuming, since the scaffolding should not appear in the final products of course (Figure 4).

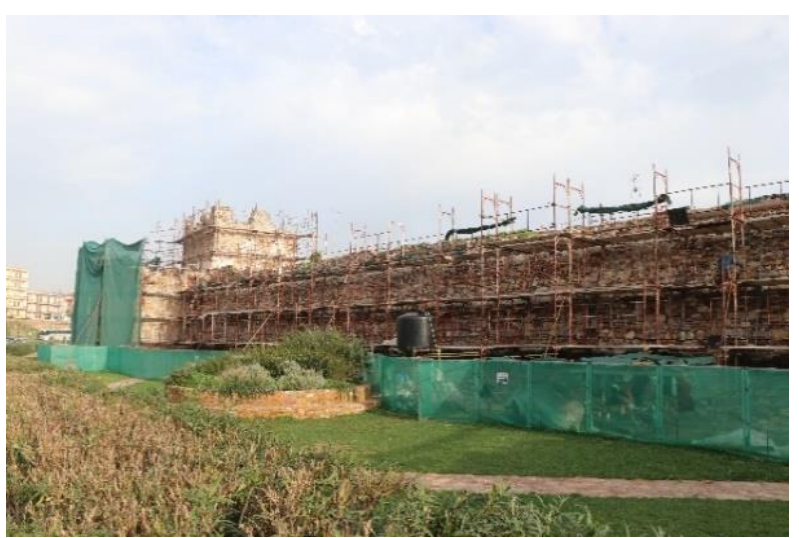

Figure 4: The part of the Fortress 
This means that during the data acquisition process the general and commonly used approach should be properly modified and adapted in order to properly image the object in a way to be able to produce the final products without the scaffolding.

Moreover, during the data processing phase any parts of the scaffolding that had been imaged should be masked out from the digital images and the 3D point cloud. Furthermore, the development of the bastion required thorough investigation as far as its geometry is concerned and the method to be used in order to get the proper and accurate product.

In order to avoid the scaffolding as much as possible, eliminate it and overcome the eventual lack of information that could arise, the bastion and the walls were scanned from ten scanner positions. In another case, fewer scans would be needed. The scaffolding could be later eliminated during the data processing and the point clouds editing procedure. The resolution of each scan was $5 \mathrm{~mm}$, in order to record all the details of the monument.

For the same reason, a large number of digital images were taken in different ways according to the part of the monument that should be documented and the difficulties that arose in each case. Before the planks of the scaffolding were removed, it was decided to use a fisheye lens $(15 \mathrm{~mm})$ and take in total 509 images standing on the planks at a very close distance (approx. $1.5 \mathrm{~m}$ ) from the object. In this way the images would not include the scaffolding and could later give the appropriate texture to the 3D model and produce the orthophotos without it. After the planks of the scaffolding were removed leaving the piping which could not be removed, a total number of 300 images with a $24 \mathrm{~mm}$ lens were taken from various distances and multiple angles.

Finally, the UAV DJI Phantom 4 Pro was used to obtain digital, aerial images for the walls, the bastion and the tower. The UAV was used in order to document the overall area of this case study and the parts of the monument that were difficult or unattainable to get digital images with the handheld camera. In total 68 images were acquired with the UAV after three flight lines at a height of $15 \mathrm{~m}$, so as to get the necessary information for the top view of the monument.

\subsection{Data Processing}

The data processing procedure could be divided in two parts, one concerning the laser scanner data and the other one concerning the digital images. The results from both these methods were combined to get the final products of the $3 \mathrm{D}$ documentation.

\subsubsection{Image Based Modelling}

The Image Based Modelling (IBM) software Agisoft Photoscan v.1.4.2 was used to produce the $3 \mathrm{D}$ detailed textured model as well as the orthophotos of planar and cylindrical surfaces. The overall process in this software, as is the case with all similar ones, involves contemporary computer vision algorithms adapted to confront the challenge of processing a huge number of images and extracting useful metric information from them.

Initially, each architectural structure was processed separately, thus forming a total of 4 groups, i.e. the images for the bastion, of the tower and of the walls acquired with the two DSLR cameras and the images for the top view of the monument acquired with the UAV. Thus, the captured images were loaded in four separate chunks, examined and evaluated, while those unnecessary, useless, blurred or badly lit ones were excluded, since they could obstruct the automated algorithm and lead to erroneous results. In addition, masks were applied on all images where the scaffolding appeared, a process extremely important to produce the orthophotos and the 3D textured model.
The next step was the automatic detection of the pre-marked targets to facilitate the alignment of the images and the scaling and georeferencing of the 3D point cloud. Additional nonartificial points were measured as markers mainly on the images of the walls, to help the alignment process and have better results in a shorter time. The alignment of images is the most important stage of the whole process, because with it the relative orientation of the images is achieved. An acceptable and reliable relative orientation ensures the production of an accurate model. Finally, the separate chunks were merged together, and their alignment was performed. This approach has enabled continuous control of the process and led to the production of correct, reliable and quality products.

Subsequently, the coordinates of each target were imported to the project in order to georeference the images and point cloud to the established reference system. Any deviations that arose had to be checked and eliminated at this step of the process. The maximum deviation should to be smaller than the allowed of $5 \mathrm{~mm}$, since the scale of 1:25 dictates the final accuracy of the products to be $6.25 \mathrm{~mm}$.

The dense point cloud was generated and subsequently extracted to be further processed with the Geomagic Wrap 2017 software, as described below.

\subsubsection{Laser Scanner 3D Point Clouds}

The registration of the scanned point clouds was achieved using the Faro Scene software, were the Cloud-to-Cloud method was applied since scans had the sufficient overlap, and the Target-toTarget method was applied to georeference the aligned scans. The registered point clouds were further processed using the Geomagic Wrap 2017 software, which delivers a very powerful toolbox to transform 3D scan data and imported files into 3D models for immediate use. Several commands are available that allow the correction of common problems with point objects. Noise reduction compensates for scanner error (noise) by moving points to statistically correct locations. Noise can make sharp edges dull and make smooth curves rough. When this command is used, the result is a smoother, less noisy arrangement of points. Uniform reduce the number of points on flat surfaces uniformly, but reduces the number of points on curved surfaces to a specified density, using the Uniform command. The Dense Point Cloud which produced with the IBM software mentioned above, was also processed in Geomagic Wrap 2017, in order to fulfill the scans and to generate the final point cloud for the whole monument. Finally, the point object was converted to a polygon object, by converting the point cloud to a mesh. This mesh was further processed using the appropriate commands and filters in order to obtain the final $3 \mathrm{~d}$ surface of the object. The last process includes filling of some inevitable holes on the surface, invertion of any inaccurate triangles and smoothing if sharp edges where necessary.

\subsubsection{D Textured Model and Orthophotos}

The final $3 \mathrm{~d}$ surface was imported into the IBM software to produce the final products so as to build the texture of the $3 \mathrm{D}$ mesh and to produce the set of the required orthophotos as well as the development of the bastion. Choosing the appropriate rendering method for the 3D textured model in each case is an important step for the optimisation and visual performance of the 3D model and its overall quality. At this step, the application of masks at the scaffolding was a crucial action in order to avoid the projection of it on the 3D surface. Furthermore, some radiometric corrections were necessary and were performed using standard image processing software. 
The orthophotos and the 2D drawings that were produced include (Figures 5 and 6):

1. The top view of the monument at scale 1:40.

2. The development of the bastion at scales 1:25 and 1:40

3. The four facades of the tower at scales $1: 25$ and 1:40

4. The façade of the walls at scales 1:25 and 1:40

5. One vertical section intersecting both the bastion and the tower in the middle

6. One vertical section intersecting the walls in the middle

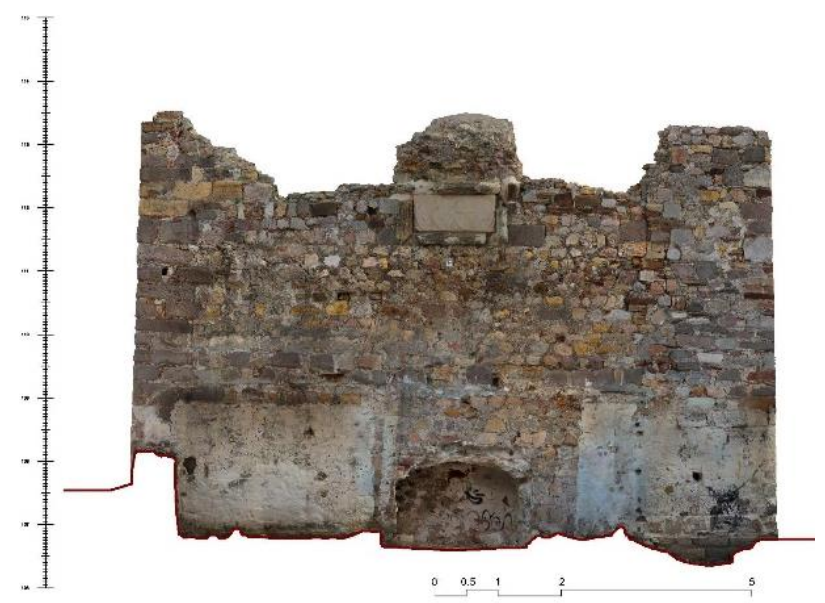

Figure 5:The orthophoto of the tower's west façade

For the generation of the orthophotos a ground pixel size of $2 \mathrm{~mm}$ was chosen, while all the necessary information and details for the section and ground lines were extracted from the threedimensional model on the specific plane each time. The architectural details and the typology of the monument played a fundamental role in the determination of the projection planes for each case, in order to avoid any geometric distortions since each architectural structure presents different inclinations, as already mentioned.

Finally, a 3D virtual tour of the detailed textured model was created in high resolution, for a more integrated visualization of the monument, to promote and enhance the restoration project and the Medieval Castle of Chios.

The development of the bastion was the challenging part of this case study, since there was the need to decide which method should be used for the process in a very short time, providing a complete, accurate and detailed result in orthophoto. The generation of sections at different levels of the bastion led to the conclusion that its surface is not entirely cylindrical but slightly conical. The deviation occurs in the upper part of the bastion where the angle of inclination was determined at 0.0006 degrees in the last $1.5-2.0$ meters. Thorough investigation led to the conclusion that if this deviation were neglected, it would result to insignificant distortion to the projected segment lengths for the required scales (1:40 and 1:25). Therefore, the bastion's surface could be processed as a cylinder, for this case.

After thorough research in different approaches and strategies that can be implemented nowadays, to produce a surface development, the following software have been tested: The open source software Cloud Compare, and the two commercial ones Geomagic Wrap 2017 and Agisoft Photoscan v.1.4. Unfortunately, Cloud Compare unrolls the 3D point cloud and produces an orthoimage from it. This results to a final resolution which is not enough for the drawings required. Moreover, Geomagic Wrap 2017 flattens complex cylindrically-wrapped geometry for better analysis and unrolls points and 3D surfaces in planar space. However, both these pieces of software do not support the production of a development and the orthoprojection of images resulting to high resolution and detailed orthophoto. This was the reason why Photoscan v.1.4 was finally chosen for the development of the bastion and the production of the orthophoto at scales 1:25 and 1:40 (Figure 7). Furthermore, it provides useful tools for selecting specific parts of images to be used for the production of the othomosaic. Photoscan offers four methods to perform the projection on a cylindrical surface, according to how the four core elements - cylinder axis and radius, zero-plane and zero-line - are defined. For this case, the axis and radius of the cylinder that was best describing the bastion, were defined after measurements and sections that were performed on the 3D surface.

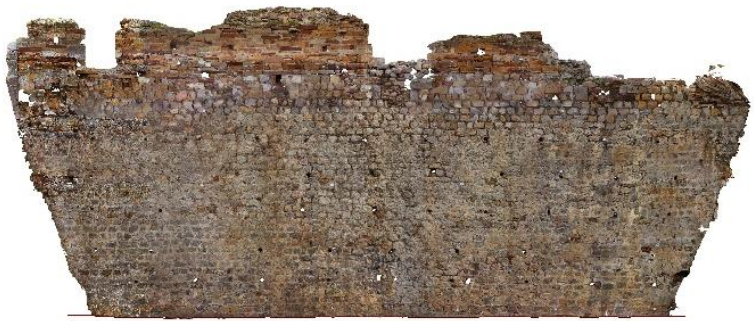

Figure 7: The development of the cylindrical bastion

The production of the required architectural vector drawings was mainly based on the orthophotos. These drawings were produced by suitably tracing the orthophotos in a CAD environment, in order to represent the masonry, the structural details and the pathology. The initial digital images were always available to be used for proper interpretation in dubious cases.

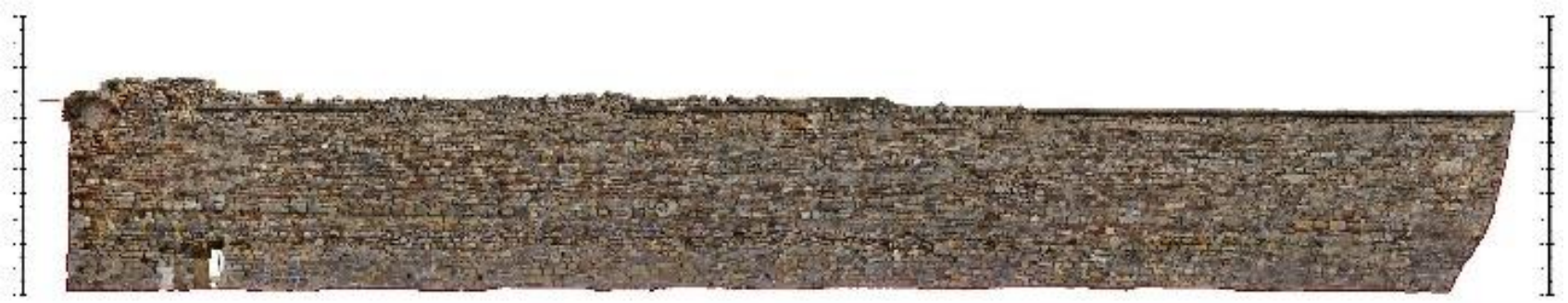

Figure 6: The orthophoto of the walls 


\section{EVALUATION OF DOCUMENTATION RESULTS}

The criteria used for the quality control of the projects' products (3D model, orthophotos) are those of conformity, completeness and accuracy.

The concept of conformity represents the degree of faithful depiction of the object, while the concept of completeness, the coverage degree of the object documented. The concept of conformity represents the degree of faithful depiction of the object, while the concept of completeness, the coverage degree of the object documented. The completeness and conformity check are performed by visual observation which entails a high degree of subjectivity.

The spatial accuracy of the 3D model was assessed by examining the reprojection error of the Ground Control Points (GCPs). Regarding the accuracy of the model, the average reprojection error of the GCPs' coordinates in the model is $\sigma_{\mathrm{xyz}}=$ $\pm 7.5 \times X X m m$, which is considered acceptable.

The accuracy of the orthophotos was assessed by examining the a posteriori standard error between the geodetic coordinates as measured in the field and their corresponding values in the produced orthophoto of the GCPs used as check points. The $a$ posteriori error was $\sigma \mathrm{x}= \pm 5.7$ and $\sigma \mathrm{y}= \pm 3.3 \mathrm{~cm}$. Considering that the GCPs are more or less uniformly distributed, the errors may be considered that express the accuracy of the points forming the entire model. Furthermore, the average GCP error can conceal possible problematic measurements.

\section{CONCLUDING REMARKS}

Close range photogrammetry is the appropriate method to produce 3D rendered models of fortification monuments. The use of aerial means, such as in this case, remains imperative. The use of SfM-MVS software yields a satisfactory 3D model in a cost beneficial way, limiting significantly the field work, but sometimes severely expanding the processing time. A thorough visual inspection of the final project and careful assessment of the data provided by the software's report is advisable. The use of automated photogrammetric and computer vision techniques through commercial software may lead to dangerous results if applied without caution. It is strongly advised to implement these techniques with deep theoretical knowledge in order to do justice both to the methodology and the object documented. All indicators of accuracy such as GCP errors, spatial resolution errors and image residuals should be reviewed along with the number of photos and GCPs, their distribution and relative position. The possibilities and effectiveness of the software available are significant. The transition from a raster product of an image-based technique, sizing a few GB, to a vector product of a few MB requires a process of deduction and careful interpretation ideally performed in cooperation with the end users of the project. The produced geometric documentation served as an indispensable base material for the restoration of the fortress of Chios (Figure 8).

\section{REFERENCES}

Almagro-Gorbea A., Orihuela Uzal A., 2015. Metodos para documentar y restaurar zonas de dificil acceso en el Castillo de Salobrena (Granada). In: Defensive Architecture of the Mediterranean XV to XVIII centuries, Vol. 1, pp. 287-290.

Chazaly B., 2001. Cylindrical Orthophotography: Complete Coverage of Inside/Outside Cylindrical Surfaces such as Castle Towers. In proceedings XVIII CIPA Symposium, 18-21 September 2001, Postdam, Germany, ISPRS Archives Volume XXXIV-5/C7.

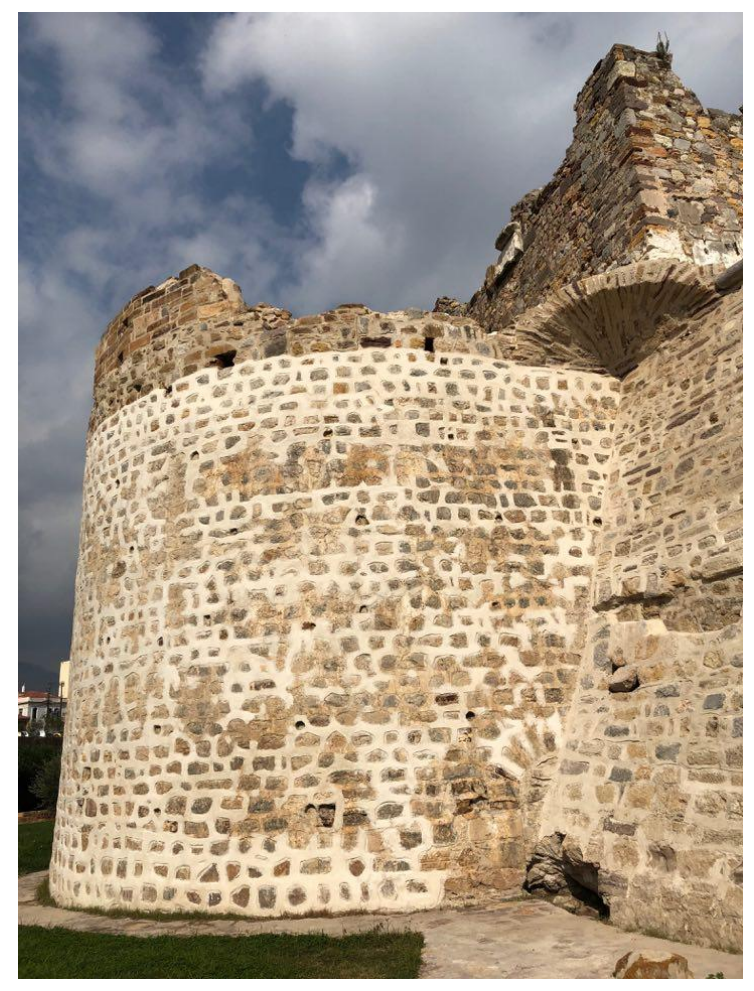

Figure 8: The bastion after restoration (http://www.chioscastle.gr)

Chiabrando F., De Bernardi M.L., Curetti S., 2009. Integration of low-cost geomatics techniques to support the architectural project the Perlo castle area survey. XXII CIPA Symposium Kyoto, Japan.

Drap P., Durand A., Seinturier J., Vannini G., Nucciotti M., 2005. Full XML documentation from photogrammetric survey to 3D visualization. The case study of Shawbak castle in Jordan. XX CIPA International Symposium Torino, Italy.

Feltham R., 1990. Determining cylindrical parameters. The Photogrammetric Record, vol. XIII (75), pp. 407-414

Georgopoulos, A., Ioannidis, C., Makris, G., Tournas, E., \& Tapinaki, S., 2002. Digitally developing works of art. In Surveying and documentation of historic buildings, monuments, sites: traditional and modern methods: Potsdam (Germany), September 18-21, 2001: proceedings of the XVIII. International Symposium, CIPA 2001, Vol. 34, pp. 277-281. Potsdam.

Gianniou, P., Georgopoulos A., Tsakiri M., Della K., 2007. The documentation of the medieval entrance of the Rhodes fortification complex. XXI International CIPA Symposium, Athens Greece.

Grussenmeyer P., Yasmine J., 2003. The restoration of Beaufort castle (South Lebanon) A 3D restitution according to historical documentation. XIX International CIPA Symposium, Antalya Turkey.

Ioannidis, C., Potsiou, C., 1999. Detailed Restitution and Representation of the Seaward Castle of Chios. Proceedings (CD) of XVII CIPA International Symposium, Recife, Brazil.

Kersten T., Mechelke K. and Maziull L., 2015. 3D model of Al Zubarah fortress in Qatar - Terrestial Laser Scanning vs Dense Image Matching. in The International Archives of the Photogrammetry, Remote Sensing and Spatial Information Sciences, Vol. XL-5/W4, pp.1-8. 
Papathanassiou-Zuhrt D., Doumi M., 2006. Creating Quality Visitor Experiences: A Best Practice Management at the Palazzo Giustiniani in Chios, Greece. Paper presented at the ICOMOS DEMHIST ANNUAL WORKSHOP 2006, Managing The Past For The Future. Sustaining Historic House Museums In The 21st Century Valetta.

Rodriguez-Navarro P., Verdiani G., 2013. Digital survey and interpretation of a fortification fragment: the Cadi bridge at the feet of the Alhambra hill, Granada. in Digital Heritage International Congress (DigitalHeritage), Vol. 2, pp. 363 - 366.

Ringle K., Nutto M., Teschauer O., Mohn C., 2005. Integration of historical plans into a modern facility management system taking the castle of Heidelberg as an example. XX CIPA International Symposium Torino, Italy.

Sammartano G., Spanò A., 2017. High Scale 3D Modelling and Orthophoto of Curved Masonries for a Multipurpose Representation, Analysis and Assessment. In the proceedings GEOMATICS \& RESTORATION- Conservation of Cultural Heritage in the Digital Era, 22-24 May 2017, Florence, Italy, ISPRS Archives, Vol. XLII-5/W1, 2017.

Tapinaki, S., Georgopoulos, A., \& Sellis, T., 2005. Design of a Database System for Geometric Documentation. In XX CIPA International Symposium - International Cooperation to Save the World's Cultural Heritage. Torino, Italy.

Theodoropoulou I., 1996. Digital Monoplotting of Cylindrical Objects. Diploma Thesis, School of Rural and Surveying Engineering, National Technical University of Athens.

Theodoropoulou I., Petsa E., Karras G. E., 2001. Digital Surface Development of Large Cylindrical and Conical Structures with a Single Image Technique. The Photogrammetric Record, 17(97), pp. 25-37.

Vrettou, F., Georgopoulos, A., 2016. Castle Penteskoufi: Geometric Documentation. Proceedings of the International Conference on Modern Age Fortifications of the Mediterranean Coast, FORTMED 2016, defensive architecture of the Mediterranean XV to XVIII centuries, Vol. IV, pp. 317-323. 